\title{
Global strong solution of the Navier-Stokes equations in 4 and 5 dimensional unbounded domains
}

by

\section{Hideo KOZONO}

Graduate School of Polymathematics Nagoya University Nagoya 464-8602 JAPAN e-mail: kozono@math.nagoya-u.ac.jp

and

\section{Hermann SOHR}

Fachbereich der Mathematik Universität Paderborn 33095 Paderborn, DEUTSCHLAND e-mail: hsohr@uni-paderborn.de

ABSTRACT. - Global strong solutions of the Navier-Stokes equations in 4 and 5 dimensional domains with non-compact boundaries are constructed. We prove also their asymptotic behaviour as $t \rightarrow \infty$.

(C) 1999 L'Association Publications de l'Institut Henri Poincaré. Published by Elsevier B.V. All rights reserved

\section{INTRODUCTION}

The purpose of this paper is to construct global strong solutions with small initial data of the Navier-Stokes equations in 4 and 5 dimensional unbounded domains. We are mainly interested in unbounded domains with non-compact boundaries. Let $\Omega$ be a domain in $\mathbf{R}^{n}(n=4,5)$ with uniformly $C^{3}$-boundary $\partial \Omega$. Our result covers the case when $\partial \Omega$ is non-compact. The motion of the incompressible fluid occupied in $\Omega$ is governed by the

2 AMS Classifications $35 \mathrm{Q} 10$

Annales de l'Institut Henri Poincaré - Analyse non linéaire - 0294-1449

Vol. 16/99/05/

(C) 1999 L'Association Publications de l'Institut Henri Poincaré. Published by Elsevier B.V. All rights reserved 
following Navier-Stokes equations:

$$
\left\{\begin{array}{l}
\frac{\partial u}{\partial t}-\Delta u+u \cdot \nabla u+\nabla p=0 \quad \text { in } x \in \Omega, t>0, \\
\operatorname{div} u=0 \quad \text { in } x \in \Omega, t>0 \\
u=0 \quad \text { on } \partial \Omega, t>0 \\
\left.u\right|_{t=0}=a,
\end{array}\right.
$$

where $u=u(x, t)=\left(u^{1}(x, t), \cdots, u^{n}(x, t)\right)$ and $p=p(x, t)$ denote the unknown velocity vector and the pressure of the fluid at point $(x, t) \in \Omega \times(0, \infty)$, while $a=a(x)=\left(a^{1}(x), \cdots, a^{n}(x)\right)$ is a given initial velocity vector field. For simplicity, we assume that the external force is equal to zero.

Since the pioneer work of Leray [20], energy decay of weak solutions to $(\mathrm{N}-\mathrm{S})$ in unbounded domains has been discussed by many authors and it is now clarified that the asymptotic behavior of strong solutions $u(t)$ as $t \rightarrow \infty$ plays an important role in such a decay problem. On account of lack compactness, the problem of existence of global strong solutions in unbounded domains seems to be more difficult than that in bounded domains; we need to pay a more exquisite attention to the analysis near $\lambda=0$ of the resolvent $(A+\lambda)^{-1}$ for the Stokes operator $A$. In recent years, a number of skillful technique such as method of compact perturbation from the whole space $\mathbf{R}^{n}$ were developed and applied to the exterior problem. $L^{p}-L^{q}$ estimates for the Stokes semigroup $\left\{e^{-t A}\right\}_{t \geq 0}$ in exterior domains are established by Giga-Sohr [11], Iwashita [14] and Borchers-Miyakawa [2] and the decay properties of weak and strong solutions are investigated to a considerable extent ([3], [17]). In unbounded domains with non-compact boundaries, however, there is no $L^{p}$-theory for the Stokes operator and the only $L^{2}$-theory is available. Based on the linear analysis of the Stokes operator, Kato [16] and Giga-Miyakawa [10] obtained a priori $L^{p}$-bounds for $p>n$ of solutions to (N-S), which yields global existence of strong solutions. On the other hand, $L^{2}$-method enables us only to get the energy inequality which bounds $\|\nabla u(t)\|_{2}$ for all $t \geq 0$ (see (E.I.) below). By the Sobolev embedding $H^{1}(\Omega) \subset L^{2 n /(n-2)}(\Omega)$, we can dominate the $L^{p}$-norm for $p>n$ of solutions if the spatial dimension $n \leq 3$. Galdi-Maremonti [8] first established apriori and decay estimates in $H^{1}$ in case $n=3$, and then Maremonti [21] improved the decay rates of weak solutions. After their results, making use of the identity $\|\nabla u\|_{2}=\left\|A^{1 / 2} u\right\|_{2}$, Kozono-Ogawa [18], [19] derived a sharp estimate of the nonlinear term $u \cdot \nabla u$ in terms of the fractional power $A^{\alpha}$ of the Stokes operator and proved the existence of global strong solutions to (N-S) in general unbounded domains in case $n=2$ and $n=3$ for large and small data, respectively. 
To treat a higher dimension, one needs to get more a priori estimates than those in $H^{1}(\Omega)$. In the present paper, we shall establish $H^{2}$-a priori bounds which yield $L^{p}$-estimates for $p>n$ of solutions even if the dimension $n=4$ and $n=5$. To this end, we shall make use of the estimate given by Heywood [12]

$$
\left\|D^{2} u\right\|_{2} \leq C\left(\|\nabla u\|_{2}+\|A u\|_{2}\right) .
$$

Recently, Borchers-Miyakawa [3] showed the existence of weak solutions $u$ of (N-S) with $\lim _{t \rightarrow \infty}\|u(t)\|_{2}=0$ for $n \leq 4$. This restriction on $n$ stems from validity of the energy inequality of the strong form

$$
\|u(t)\|_{2}^{2}+2 \int_{s}^{t}\|\nabla u(\tau)\|_{2}^{2} d \tau \leq\|u(s)\|_{2}^{2}
$$

for all $s$ and $t$ such that $0 \leq s \leq t$. The importance of the above inequality (E.I.) in unbounded domains was pointed out by Masuda [23] and Kato [16]. Leray [20] called a weak solution $u$ satisfying (E.I.) a turbulent solution, the existence of which was shown for $n \leq 4$ up to the present(see Kato [16] and Miyakawa-Sohr [25]). Our global strong solution satisfies (E.I.) and hence we can construct the solution decaying in $L^{2}$ even for $n=5$. Moreover, we shall show a sharp decay of the strong solution as $t \rightarrow \infty$, which seems to be optimal. The decay rates will be furnished in terms of the fractional powers $A^{\alpha}$ for $0 \leq \alpha \leq 1$. Since we need the additional term $\|\nabla u\|_{2}$ on the right hand side of the above Heywood's estimate, the decay $\left\|A^{\alpha} u(t)\right\|_{2}$ for $1 / 2<\alpha \leq 1$ cannot be directly obtained by investigating the linearized equation of (N-S). To get around this difficulty, we shall first establish the decay of $\frac{\partial u}{\partial t}(t)$ as $t \rightarrow \infty$. Such a method was first introduced by Masuda [22]. To get sharper decay, however, we need more calculations than [22].

In Section 1 we shall state our main theorem. Section 2 is devoted to the estimate of the nonlinear term $u \cdot \nabla u$ by $(A+\varepsilon)^{-1}$ which holds independently of $\varepsilon>0$. To this end, we shall first introduce the Hilbert scale via fractional powers $A^{\alpha}$ and define a family of certain homogeneous Sobolev spaces by complex interpolation. This procedure is done by Miyakawa [24] in 3dimensional exterior domains. Then we shall apply Heywood's estimate to obtain bounds of higher derivatives. In Section 3, an explicit representation of the time interval where the strong solutions exist will be given in terms of the Hilbert scale of the initial data and we shall show the a priori estimate of solutions in such a scale. As a result the global strong solution will be constructed provided the initial data $a$ is sufficiently small. Finally in Section 4, we shall show the decay property of the strong solution. 


\section{RESULT}

Throughout this paper, we impose the following assumption on the domain $\Omega$.

Assumption. - $\Omega\left(\subset \mathbf{R}^{n}, n=4,5\right)$ is of class uniformly $C^{3}$ (for the definition, see Tanabe [28, Definition 1.2.2]) and regular, i.e., the boundary $\partial \Omega$ consists of finitely many, disjoint simple $C^{3}$ surfaces.

We may treat the domain $\Omega$ with a non-compact boundary $\partial \Omega$.

Before stating our results, we introduce some notations and function spaces. Let $C_{0, \sigma}^{\infty}$ denote the set of all $C^{\infty}$ vector functions $\phi=\left(\phi^{1}, \cdots, \phi^{n}\right)$ with compact support in $\Omega$, such that div $\phi=0 . L_{\sigma}^{2}$ is the closure of $C_{0, \sigma}^{\infty}$ with respect to the $L^{2}$-norm \|\|$_{2} ;(\cdot, \cdot)$ denotes the $L^{2}$-inner product. $\|\cdot\|_{p}$ is the $L^{p}$-norm on $\Omega, 1 \leq p \leq \infty$. For simplicity, we abbreviate $\|\cdot\|_{2}=\|\cdot\| \cdot H_{0, \sigma}^{1}$ denotes the closure of $C_{0, \sigma}^{\infty}$ with respect to the norm

$$
\|\phi\|_{H^{1}}=\|\phi\|+\|\nabla \phi\|,
$$

where $\nabla \phi=\left(\partial \phi^{i} / \partial x_{j} ; \quad i, j=1, \cdots, n\right)$. When $X$ is a Banach space, we denote by $\|\cdot\|_{X}$ the norm on $X$. Then $C^{m}\left(\left(t_{1}, t_{2}\right) ; X\right)$ and $L^{p}\left(\left(t_{1}, t_{2}\right) ; X\right)$ are the usual Banach spaces, where $m=0,1,2, \cdots$ and $t_{1}$ and $t_{2}$ are real numbers such that $t_{1}<t_{2} \leq \infty$. Further, $B C\left(\left(t_{1}, t_{2}\right) ; X\right)$ is the set of all functions $u$ in $C^{0}\left(\left(t_{1}, t_{2}\right) ; X\right)$ such that $\sup _{t_{1}<t<t_{2}}\|u(t)\|_{X}<\infty$.

We denote by $P$ the orthogonal projection from $L^{2}(\Omega)$ onto $L_{\sigma}^{2}$. Then the Stokes operator $A$ is defined by $A=-P \Delta$ with domain $D(A)=\left\{u \in H^{2}(\Omega) ;\left.u\right|_{\partial \Omega}=0\right\} \cap L_{\sigma}^{2}$. More precisely; $A$ coincides with the non-negative self-adjoint operator defined by the quadratic form $a(u, v)=(\nabla u, \nabla v), u, v \in H_{0, \sigma}^{1}$, that is, $A u=f \quad\left(u \in D(A), f \in L_{\sigma}^{2}\right)$ is equivalent to the relation $a(u, v)=(f, v)$ for all $v \in H_{0, \sigma}^{1}$. Therefore we have $D\left(A^{1 / 2}\right)=H_{0, \sigma}^{1}$ and

$$
\left\|A^{1 / 2} u\right\|=\|\nabla u\|, \quad u \in D\left(A^{1 / 2}\right) .
$$

Let $0<\alpha \leq 1 . D\left(A^{\alpha}\right)$ is a Banach space equipped with the graph norm

$$
\|u\|_{D\left(A^{\alpha}\right)}=\|u\|+\left\|A^{\alpha} u\right\| .
$$

Then it follows from Fujita-Morimoto [6] that

$$
D\left(A^{\alpha}\right) \subset H^{2 \alpha, 2} \quad \text { (continuous embedding), }
$$

where $H^{2 \alpha, 2}$ denotes the Sobolev space over $\Omega$. 
Applying the orthogonal projection $P$ to both sides of (N-S), we get the following differential equation in $L_{\sigma}^{2}$ :

$$
\left\{\begin{array}{l}
\frac{d u}{d t}+A u+P(u \cdot \nabla u)=0, \quad 0<t<\infty \\
u(0)=a
\end{array}\right.
$$

Our result on global strong solutions now reads:

THEOREM. - Let $n=4$ and 5. There is a constant $\mu=\mu(n)>0$ such that if $a \in D\left(A^{\frac{n}{4}-\frac{1}{2}}\right)$ satisfies $\|a\|_{D\left(A^{\frac{n}{4}-\frac{1}{2}}\right)} \leq \mu$, then we have a unique strong solution $u$ of $(E)$ with the following properties:

$$
\begin{aligned}
& u \in B C\left([0, \infty) ; D\left(A^{\frac{n}{4}-\frac{1}{2}}\right)\right) \cap C((0, \infty) ; D(A)) \\
& \frac{d u}{d t} \in C\left((0, \infty) ; D\left(A^{\frac{1}{2}}\right)\right)
\end{aligned}
$$

the energy identity

$$
\|u(t)\|^{2}+2 \int_{0}^{t}\|\nabla u(\tau)\|^{2} d \tau=\|a\|^{2}
$$

holds for all $t \geq 0$. Moreover, such a solution $u$ decays like

$$
\begin{aligned}
& \left\|A^{\alpha} u(t)\right\|=o\left(t^{-\alpha}\right) \quad \text { for } 0 \leq \alpha \leq 1, \\
& \left\|\frac{d u(t)}{d t}\right\|=o\left(t^{-1}\right)
\end{aligned}
$$

as $t \rightarrow \infty$.

Remark. - In case when $\Omega=\mathbf{R}^{n}$, the half space $\mathbf{R}_{+}^{n}$, a bounded domain or an exterior domain with compact boundaries, the global strong solution of (E) was obtained provided $a$ is sufficiently small in $L^{n}(\Omega)$. See Kato [16], Ukai [29], Giga-Miyakawa [10] and Iwashita [14]. It should be noted that, by (1.2) the space $D\left(A^{\frac{n}{4}-\frac{1}{2}}\right)$ is continuously embedded into $L^{n}(\Omega)$. In general unbounded domains $\Omega \subset \mathbf{R}^{3}$ with non-compact boundaries $\partial \Omega$, Kozono-Ogawa [19] obtaincd the same result as in the above theorem under the weaker assumption that $\left\|A^{\frac{1}{4}} a\right\|$ is small; $\|a\|$ itself need not be small. In our theorem, however, $\|a\|$ must be also small, which stems from the fact that $\|a\|_{p}$ for $p>n$ does not seem to be dominated only by the homogeneous norm $\left\|A^{\alpha} a\right\|$ with $\alpha=\frac{n}{2}\left(\frac{1}{2}-\frac{1}{p}\right)$ (see [19, Lemma 2.1]). 


\section{PRELIMINARIES}

In this section, we shall estimate the nonlinear term $u \cdot \nabla u$ in terms of the fractional power $A^{\alpha}$. In what follows we shall denote by $C$ various constants. In particular, $C=C(*, \cdots, *)$ denotes constants depending only on the quantities appearing in the parenthesis. Let us first introduce the following estimate given by Heywood [12, Lemma 6]:

I.FMMA 2.1 (HeywoOd). - Under our assumption on the domain $\Omega$, there is a constant $C=C(\Omega)$ such that

$$
\left\|D^{2} u\right\| \leq C(\|\nabla u\|+\|A u\|) \quad \text { for all } u \in D(A) .
$$

where $D^{2} u=\left(\frac{\partial^{2} u}{\partial x^{i} \partial x^{j}}\right)_{i, j=1, \cdots, n}$.

It should be noted that in general we cannot avoid to add $\|\nabla u\|$ on the right hand side of (2.1)(see Borchers-Miyakawa [4] and Kozono-Ogawa [17]). We shall next define the space $\widehat{D}\left(A^{\alpha}\right)$ for $0 \leq \alpha \leq 1$ as follows.

For $0 \leq \alpha \leq 1 / 2$

(2.2) $\widehat{D}\left(A^{\alpha}\right)=$ the completion of $D(A)$ with respect to the norm $\left\|A^{\alpha} u\right\|$.

For $1 / 2 \leq \alpha \leq 1$

$\widehat{D}\left(A^{\alpha}\right)$

(2.3) $=$ the completion of $D(A)$ with respect to the norm $\left\|A^{1 / 2} u\right\|+\left\|A^{\alpha} u\right\|$.

Note that $\widehat{D}\left(A^{\alpha}\right)$ is larger than $D\left(A^{\alpha}\right)$. Then it follows from Miyakawa [24, Theorems 2.5-2.5] that

LEMMA 2.2 (Miyakawa).

$$
\begin{aligned}
{\left[L_{\sigma}^{2}, \widehat{D}\left(A^{\frac{1}{2}}\right)\right]_{\alpha} } & =\widehat{D}\left(A^{\frac{\alpha}{2}}\right), \\
{\left[\widehat{D}\left(A^{\frac{1}{2}}\right), \widehat{D}(A)\right]_{\alpha} } & =\widehat{D}\left(A^{\frac{1}{2}+\frac{\alpha}{2}}\right)
\end{aligned}
$$

for $0 \leq \alpha \leq 1$, where $[X, Y]_{\alpha}$ denotes the space of complex interpolation between $X$ and $Y$.

By Lemmata 2.1 and 2.2, we can establish the following estimate of the nonlinear term $u \cdot \nabla u$ : 
LeMma 2.3. - Let $n=4$ and $n=5$. For $n / 2-2 \leq \theta<1$, there is $a$ constant $C=C(n, \theta)$ such that

$$
\begin{array}{r}
\|P(u \cdot \nabla v)\| \leq C\left(\left\|A^{\frac{1}{2}} u\right\|+\left\|A^{\frac{1}{2}+\frac{\theta}{2}} u\right\|\right)\left(\left\|A^{\frac{1}{2}} v\right\|+\left\|A^{\frac{n}{4}-\frac{\theta}{2}} v\right\|\right) \\
\left\|(A+\varepsilon)^{-\frac{\theta}{2}} P(u \cdot \nabla v)\right\| \leq C\left\|A^{\frac{1}{2}} u\right\|\left(\left\|A^{\frac{1}{2}} v\right\|+\left\|A^{\frac{n}{4}-\frac{\theta}{2}} v\right\|\right)
\end{array}
$$

for all $u \in D\left(A^{\frac{1}{2}+\frac{\theta}{2}}\right), v \in D\left(A^{\frac{n}{4}-\frac{\theta}{2}}\right)$ and all $\varepsilon>0$.

Proof. - Let us first show that $u \cdot \nabla v \in L^{2}$ provided $u \in D\left(A^{\frac{1}{2}+\frac{\theta}{2}}\right)$ and $v \in D\left(A^{\frac{n}{4}-\frac{\theta}{2}}\right)$. Indeed, by (1.2) and the Sobolev inequality we have the following continuous embeddings:

$$
\begin{aligned}
& D\left(A^{\frac{1}{2}+\frac{\theta}{2}}\right) \subset H^{1+\theta, 2} \subset L^{p} \text { for } \frac{1}{p}=\frac{1}{2}-\frac{1+\theta}{n}, \\
& D\left(A^{\frac{n}{4}-\frac{\theta}{2}}\right) \subset H^{\frac{n}{2}-\theta, 2} \subset H^{1, q} \text { for } \frac{1}{q}=\frac{1+\theta}{n} .
\end{aligned}
$$

Since $1 / p+1 / q=1 / 2$, the Hölder inequality yields $u \cdot \nabla v \in L^{2}$ and hence $P(u \cdot \nabla v) \in L_{\sigma}^{2}$ is well-defined. We shall next show the continuous embedding

$$
\widehat{D}\left(A^{\frac{1}{2}+\frac{\alpha}{2}}\right) \subset L^{p}, \quad 0 \leq \alpha<1 \quad \text { for } \frac{1}{p}=\frac{1}{2}-\frac{1+\alpha}{n} .
$$

By the Sobolev inequality, (1.1) and Lemma 2.1, there holds

$$
\begin{array}{r}
\|u\|_{\frac{2 n}{n-2}} \leq C\|\nabla u\|=C\left\|A^{\frac{1}{2}} u\right\| \quad \text { for } n=4,5 \\
\|u\|_{B M O} \leq C\|\nabla u\|_{4} \leq C(\|\nabla u\|+\|\nabla(\nabla u)\|) \\
\leq C\left(\left\|A^{\frac{1}{2}} u\right\|+\|A u\|\right) \text { for } n=4 \\
\|u\|_{10} \leq C\|\nabla u\|_{\frac{10}{3}} \leq C(\|\nabla u\|+\|\nabla(\nabla u)\|) \\
\leq C\left(\left\|A^{\frac{1}{2}} u\right\|+\|A u\|\right) \text { for } n=5
\end{array}
$$

for all $u \in D(A)$ with $C=C(n)$, where $B M O$ denotes the space of bounded mean oscillation over $\Omega$. It should be noted that we do not need to add $\|u\|_{4}$ and $\|u\|_{\frac{10}{3}}$ on the right hand side of the above estimates (2.9) and (2.10), respectively, since $u=0$ on $\partial \Omega$. This implies that the injection $i$ is a continuous operator as

$$
\begin{aligned}
& i: \widehat{D}\left(A^{\frac{1}{2}}\right) \hookrightarrow L^{\frac{2 n}{n-2}} \\
& i: \widehat{D}(A) \hookrightarrow\left\{\begin{aligned}
B M O, & \text { for } n=4 \\
L^{10}, & \text { for } n=5 .
\end{aligned}\right.
\end{aligned}
$$

Vol. 16, n 5-1999. 
Since $\left[L^{p}, B M O\right]_{\alpha}=L^{p /(1-\alpha)}$ for $0 \leq \alpha<1$ (see e.g., JansonJones [15]), Lemma 2.2 and the interpolation theory yield the continuous embedding (2.8). Moreover, (2.9), (2.10) and (2.11) show that the gradient $\nabla$ defines a bounded operator as

$$
\begin{aligned}
& \nabla: \widehat{D}\left(A^{\frac{1}{2}}\right) \rightarrow L^{2}, \\
& \nabla: \hat{D}(A) \rightarrow L^{\frac{2 n}{n-2}},
\end{aligned}
$$

from which and (2.5) we obtain

$$
\nabla: \widehat{D}\left(A^{\frac{1}{2}+\frac{\alpha}{2}}\right) \rightarrow L^{q}, \quad 0 \leq \alpha \leq 1 \quad \text { for } \frac{1}{q}=\frac{1}{2}-\frac{\alpha}{n} .
$$

Now let us take $p$ and $q$ in such a way that

$$
\frac{1}{p}=\frac{1}{2}-\frac{1+\theta}{n}, \quad \frac{1}{q}=\frac{1+\theta}{n} .
$$

Then by (2.8), we have

$$
\|u\|_{p} \leq C\left(\left\|A^{\frac{1}{2}} u\right\|+\left\|A^{\frac{1}{2}+\frac{\theta}{2}} u\right\|\right) \quad \text { for all } u \in D\left(A^{\frac{1}{2}+\frac{\theta}{2}}\right) .
$$

Taking $\alpha=n / 2-1-\theta$, we have by the assumption on $\theta$ and (2.13) that $0<\alpha \leq 1$ and $1 / q=1 / 2-\alpha / n$. Hence (2.12) yields

$$
\|\nabla v\|_{q} \leq C\left(\left\|A^{\frac{1}{2}} v\right\|+\left\|A^{\frac{1}{2}+\frac{u}{2}} v\right\|\right) \leq C\left(\left\|A^{\frac{1}{2}} v\right\|+\left\|A^{\frac{\mu}{4}-\frac{\theta}{2}} v\right\|\right)
$$

for all $v \in D\left(A^{\frac{n}{4}-\frac{\theta}{2}}\right)$. Since $1 / p+1 / q=1 / 2$, we have by (2.14), (2.15) and the Hölder inequality that

$$
\begin{aligned}
\|P(u \cdot \nabla v)\| & \leq\|u\|_{p}\|\nabla v\|_{q} \\
& \leq C\left(\left\|A^{\frac{1}{2}} u\right\|+\left\|A^{\frac{1}{2}+\frac{\theta}{2}} u\right\|\right)\left(\left\|A^{\frac{1}{2}} v\right\|+\left\|A^{\frac{n}{4}-\frac{\theta}{2}} v\right\|\right)
\end{aligned}
$$

for all $u \in D\left(A^{\frac{1}{2}+\frac{\theta}{2}}\right)$ and all $v \in D\left(A^{\frac{n}{4}-\frac{\theta}{2}}\right)$. This implies (2.6).

We shall next prove (2.7). By (2.4), (2.9) and the interpolation inequality, there holds a continuous inclusion

$$
\widehat{D}\left(A^{\frac{\theta}{2}}\right) \subset L^{p} \quad \text { for } \frac{1}{p}=\frac{1}{2}-\frac{\theta}{n} .
$$

Hence it follows from (2.9), (2.15) and (2.16) that

$$
\begin{aligned}
\left|\left((A+\varepsilon)^{-\frac{\theta}{2}} P(u \cdot \nabla v), \phi\right)\right| & =\left|\left(P(u \cdot \nabla v),(A+\varepsilon)^{-\frac{\theta}{2}} \phi\right)\right| \\
& \leq\|u \cdot \nabla v\|_{p^{\prime}}\left\|(A+\varepsilon)^{-\frac{\theta}{2}} \phi\right\|_{p} \\
& \leq C\|u\|_{\frac{2 u}{n-2}}\|\nabla v\|_{\frac{n}{1+\theta}}\left\|A^{\frac{\theta}{2}}(A+\varepsilon)^{-\frac{\theta}{2}} \phi\right\| \\
& \leq C\left\|A^{\frac{1}{2}} u\right\|\left(\left\|A^{\frac{1}{2}} v\right\|+\left\|A^{\frac{n}{4}-\frac{\theta}{2}} v\right\|\right)\|\phi\|
\end{aligned}
$$


for all $\phi \in C_{0, \sigma}^{\infty}$ and all $\varepsilon>0$ with $C=C(n, \theta)$. Now (2.7) follows from the duality argument. This completes the proof of Lemma 2.3.

By Lemma 2.3 we can define the weak limit $(A+\varepsilon)^{-\frac{\theta}{2}} P(u \cdot \nabla v)$ in $L_{\sigma}^{2}$ as $\varepsilon \downarrow+0$.

LEMMA 2.4. - Let $n=4,5$ and $n / 2-2 \leq \theta<1$.

(1) For $u, v \in D\left(A^{\frac{1}{2}+\frac{\theta}{2}}\right) \cap D\left(A^{\frac{n}{4}-\frac{\theta}{2}}\right)$, there exists a weak limit

$$
w-\lim _{\varepsilon \downarrow+0}(A+\varepsilon)^{-\frac{\theta}{2}} P(u \cdot \nabla v) \text { in } L_{\sigma}^{2} .
$$

(2) Let us denote by $\widetilde{F}_{\theta}(u, v)$ the weak limit:

$$
\widetilde{F}_{\theta}(u, v) \equiv w-\lim _{\varepsilon \downarrow+0}(A+\varepsilon)^{-\frac{\theta}{2}} P(u \cdot \nabla v) .
$$

Then there holds

$$
\left\|\widetilde{F}_{\theta}(u, v)\right\| \leq C\left\|A^{\frac{1}{2}} u\right\|\left(\left\|A^{\frac{1}{2}} v\right\|+\left\|A^{\frac{n}{4}-\frac{\theta}{2}} v\right\|\right)
$$

with $C=C(n, \theta)$ independent of $u$ and $v$.

Proof. - By (1.1) zero is not an eigenvalue of $A$. Hence the range $R\left(A^{\frac{\theta}{2}}\right)$ of $A^{\frac{\theta}{2}}$ is dense in $L_{\sigma}^{2}$ and we have $(A+\varepsilon)^{-\frac{\theta}{2}} A^{\frac{\theta}{2}} \phi \rightarrow \phi$ in $L_{\sigma}^{2}$ for all $\phi \in D\left(A^{\frac{\theta}{2}}\right)$ as $\varepsilon \downarrow+0$. Then for every $u, v \in D\left(A^{\frac{1}{2}+\frac{\theta}{2}}\right) \cap D\left(A^{\frac{n}{4}-\frac{\theta}{2}}\right)$ and every $\phi \in D\left(A^{\frac{\theta}{2}}\right)$, there holds

$$
\begin{aligned}
\left((A+\varepsilon)^{-\frac{\theta}{2}} P(u \cdot \nabla v), A^{\frac{\theta}{2}} \phi\right) & =\left(P(u \cdot \nabla v),\left((A+\varepsilon)^{-\frac{\theta}{2}} A^{\frac{\theta}{2}} \phi\right)\right. \\
& \rightarrow(P(u \cdot \nabla v), \phi) \quad \text { as } \varepsilon \downarrow+0
\end{aligned}
$$

Since $R\left(A^{\frac{\theta}{2}}\right)$ is dense in $L_{\sigma}^{2}$, this convergence together with (2.7) yields a weak limit $w-\lim _{\varepsilon \downarrow+0}(A+\varepsilon)^{-\frac{\theta}{2}} P(u \cdot \nabla v)$ in $L_{\sigma}^{2}$. Then the assertion (2) is an immediate consequence of (2.7) and the resonance theorem.

Let us now define an operator $F_{\theta}(u, v)$ in a rigorous way which is formally written as $A^{-\frac{\theta}{2}} P(u \cdot \nabla v)$. We shall follow the procedure of Masuda [22]. Let $\theta$ be as in the hypotheses of Lemma 2.4. Since $D\left(A^{\frac{1}{2}+\frac{\theta}{2}}\right) \cap D\left(A^{\frac{n}{4}-\frac{\theta}{2}}\right)$ is dense in $D\left(A^{\frac{n}{4}-\frac{\theta}{2}}\right)$, we can define a bilinear operator $F_{\theta}(u, v)$ for $u, v \in D\left(A^{\frac{n}{4}-\frac{\theta}{2}}\right)$ by

$$
F_{\theta}(u, v)=\mathrm{s}-\lim _{j \rightarrow \infty} \widetilde{F}_{\theta}\left(u_{j}, v_{j}\right) \quad \text { (strong limit in } L_{\sigma}^{2} \text { ), }
$$

where $\left\{u_{j}\right\}_{j=1}^{\infty}$ and $\left\{v_{j}\right\}_{j=1}^{\infty}$ are sequences in $D\left(A^{\frac{1}{2}+\frac{\theta}{2}}\right) \cap D\left(A^{\frac{n}{4}-\frac{\theta}{2}}\right)$ such that $u_{j} \rightarrow u, v_{j} \rightarrow v$ in $D\left(A^{\frac{n}{4}-\frac{\theta}{2}}\right)$. Then we have

Vol. 16, $n^{\circ} 5-1999$. 
LEMMA 2.5. - Let $n=4,5$ and $n / 2-2 \leq \theta<1$.

(1) The estimate

$$
\left\|F_{\theta}^{\prime}(u, v)\right\| \leq C^{v}\left\|A^{\frac{1}{2}} u\right\|\left(\left\|A^{\frac{1}{2}} v\right\|+\left\|A^{\frac{n}{4}-\frac{\theta}{2}} v\right\|\right)
$$

holds for all $u, v \in D\left(A^{\frac{n}{4}-\frac{\theta}{2}}\right)$ with the same constant $C=C(n, \theta)$ as in (2.17).

(2) If $u, v \in D\left(A^{\frac{1}{2}+\frac{\theta}{2}}\right) \cap D\left(A^{\frac{n}{4}-\frac{\theta}{2}}\right)$, then we have $F_{\theta}(u, v) \in D\left(A^{\frac{0}{2}}\right)$ with $A^{\frac{\theta}{2}} F_{\theta}(u, v)=P(u \cdot \nabla v)$.

Proof. - The assertion (1) can be obtained directly from (2.17) and the definition of $F_{\theta}(u, v)$. Let us prove the assertion (2). For $u, v \in$ $D\left(A^{\frac{1}{2}+\frac{\theta}{2}}\right) \cap D\left(A^{\frac{n}{4}-\frac{\theta}{2}}\right)$, we have $F_{\theta}(u, v)=\widetilde{F}_{\theta}(u, v)$. Hence by $(2.18)$,

$$
\begin{aligned}
\left(F_{\theta}(u, v), A^{\frac{\theta}{2}} \phi\right) & =\left(\widetilde{F}_{\theta}(u, v), A^{\frac{\theta}{2}} \phi\right) \\
& -\lim _{\varepsilon \downarrow+0}\left((A+\varepsilon)^{-\frac{\theta}{2}} P(u \cdot \nabla v), A^{\frac{\theta}{2}} \phi\right) \\
& =(P(u \cdot \nabla v), \phi)
\end{aligned}
$$

holds for all $\phi \in D\left(A^{\frac{\theta}{2}}\right)$, which implies the desired assertion.

Remark 2.6. - If $n / 2-2 \leq \theta \leq n / 4-1 / 2$, then we have $1 / 2+\theta / 2 \leq$ $n / 4-\theta / 2$ and the inclusion $D\left(\bar{A}^{\frac{n}{4}-\frac{\theta}{2}}\right) \subset D\left(A^{\frac{1}{2}+\frac{\theta}{2}}\right)$ holds. In such case, the above lemma states $F_{\theta}(u, v) \in D\left(A^{\frac{\theta}{2}}\right)$ with $A^{\frac{\theta}{2}} F_{\theta}(u, v)=P(u \cdot \nabla v)$ for $u, v \in D\left(A^{\frac{n}{4}-\frac{\theta}{2}}\right)$.

\section{LOCAL AND GLOBAL STRONG SOLUTIONS}

In this section, we shall give an explicit characterization of time interval $(0, T)$ while the strong solution exists, and then establish a priori estimates to show $T=\infty$ provided the initial data is small.

THeOrem 3.1. - (1) Let $n=1,5$ and let $n / 1-1 / 2<\gamma \leq(n+2) / 8$. Then for every $a \in D\left(A^{\gamma}\right)$, there exist $0<T \leq 1$ and a unique solution $u$ of $(E)$ on $(0, T)$ with

$$
\begin{aligned}
& u \in B C\left([0, T) ; D\left(A^{\gamma}\right)\right) \cap C((0, T) ; D(A)) ; \\
& \frac{d u}{d t} \in C\left((0, T) ; D\left(A^{\frac{1}{2}}\right)\right) ;
\end{aligned}
$$


the energy identity

$$
\|u(t)\|^{2}+2 \int_{0}^{t}\|\nabla u(\tau)\|^{2} d \tau=\|a\|^{2}
$$

holds for all $t \in[0, T)$. Here $T$ is estimated from below as

$$
T \geq C\left(\|a\|+\left\|A^{\gamma} a\right\|\right)^{-\frac{1}{\gamma-\left(\frac{n}{4}-\frac{1}{2}\right)}}
$$

with $C=C(n, \gamma)$ independent of a.

(2) For $n, \gamma$ as above (1) and any $\varepsilon>0$, there is a constant $\mu=\mu(n, \gamma, \varepsilon)>0$ such that if $a \in D\left(A^{\frac{n}{4}-\frac{1}{2}}\right)$ satisfies

$$
\|a\|+\left\|A^{\frac{n}{4}-\frac{1}{2}} a\right\| \leq \mu,
$$

then we have a unique solution $u$ of $(E)$ on $(0,1)$ with $(3.1)$ replaced by

$$
u \in B C\left([0,1) ; D\left(A^{\frac{n_{2}}{4}-\frac{1}{2}}\right)\right) \cap C((0,1) ; D(A))
$$

and (3.2), (3.3) for $T=1$ such that

$$
\sup _{0<t<1}\|u(t)\|+\sup _{0<t<1} t^{\gamma-\left(\frac{n}{4}-\frac{1}{2}\right)}\left\|A^{\gamma} u(t)\right\|<\varepsilon
$$

Proof. - (1) Since this theorem is concerned with the local existence of strong solutions, the proof can be done in the same away as Fujita-Kato [5] and Giga-Miyakawa [10]. However, we give the outline of the proof for completeness. Let us transform the unknown function $u(t)$ of $(\mathrm{E})$ into $v(t)$ by $u(t)=e^{t} v(t)$, which yields

$$
\left\{\begin{array}{l}
\frac{d v}{d t}+\widetilde{A} v+e^{t} P(v \cdot \nabla v)=0, \quad t>0 \\
v(0)=a
\end{array}\right.
$$

where $\widetilde{A}=A+1$. By Duhamel's principle we may solve the following integral equation

$$
v(t)=e^{-t \widetilde{A}} a-\int_{0}^{t} e^{-(t-s) \widetilde{A}} e^{s} P(v \cdot \nabla v)(s) d s .
$$

The solution $v(t)$ of $(3.8)$ is constructed by the successive approximation:

$$
\left\{\begin{array}{l}
v_{0}(t)=e^{t \tilde{A}} a \\
v_{m+1}(t)=v_{0}(t)-\int_{0}^{t} e^{-(t-s) \tilde{A}} e^{s} P\left(v_{m} \cdot \nabla v_{m}\right)(s) d s, m=0,1, \cdots
\end{array}\right.
$$


Let us recall the estimate given by Inoue-Wakimoto [13, Lemma 4.2] and Giga-Miyakawa[10, Lemma 2.2]:

$$
\|P(v \cdot \nabla w)\| \leq C\left\|\tilde{A}^{\theta} v\right\|\left\|\tilde{A}^{\gamma} w\right\|, \quad v \in D\left(\Lambda^{\theta}\right), w \in D\left(\Lambda^{\gamma}\right)
$$

for $\theta=(n+2) / 4-\gamma$ with $C=C(n, \gamma)$. This estimate can be proved in a similar way as for Lemma 2.3. Since $n / 4-1 / 2<\gamma \leq(n+2) / 8$, we have

$$
\frac{n}{4}-\frac{1}{2}<\theta<1
$$

Then for $n / 4-1 / 2 \leq \alpha<1$ and $T>0$, there holds

$$
K_{\alpha, m}(T) \equiv \sup _{0<t<T} t^{\alpha-\left(\frac{n}{4}-\frac{1}{2}\right)}\left\|\widetilde{A}^{\alpha} v_{m}(t)\right\|<\infty, \quad m=0,1, \cdots
$$

Indeed, for $m=0$, we have

$$
\begin{aligned}
t^{\alpha-\left(\frac{n}{4}-\frac{1}{2}\right)}\left\|\widetilde{A}^{\alpha} v_{0}(t)\right\| & =t^{\alpha-\left(\frac{n}{4}-\frac{1}{2}\right)}\left\|\widetilde{A}^{\alpha} e^{-t \widetilde{A}} a\right\| \\
& =t^{\alpha-\left(\frac{n}{4}-\frac{1}{2}\right)}\left\|\widetilde{A}^{\alpha-\left(\frac{n}{4}-\frac{1}{2}\right)} e^{-t \widetilde{A}} \widetilde{A}^{\frac{n}{4}-\frac{1}{2}} a\right\|
\end{aligned}
$$

for all $t>0$ and $n / 4-1 / 2 \leq \alpha<1 . K_{\alpha, 0}(T)$ can be defined as

$$
K_{\alpha, 0}(T) \equiv \sup _{0<t<T} t^{\alpha-\left(\frac{n}{4}-\frac{1}{2}\right)}\left\|\widetilde{A}^{\alpha} e^{-t \widetilde{A}} a\right\|, \quad \frac{n}{4}-\frac{1}{2} \leq \alpha<1 .
$$

Suppose that (3.12) is true for $m$. Then it follows from (3.10) that

$$
\begin{aligned}
& \left\|\widetilde{A}^{\alpha} v_{m+1}(t)\right\| \\
& \leq\left\|\widetilde{A}^{\alpha} v_{0}(t)\right\|+\int_{0}^{t}\left\|\widetilde{A}^{\alpha} e^{-(t-s) \widetilde{A}} e^{s} P\left(v_{m} \cdot \nabla v_{m}\right)(s)\right\| d s \\
& \leq K_{\alpha, 0}(T) t^{\frac{n}{4}-\frac{1}{2}-\alpha}+C e \int_{0}^{t}(t-s)^{-\alpha}\left\|\widetilde{A}^{\theta} v_{m}(s)\right\|\left\|\widetilde{A}^{\gamma} v_{m}(s)\right\| d s \\
& \leq K_{\alpha, 0}(T) t^{\frac{n}{4}-\frac{1}{2}-\alpha}+C e K_{\theta, m}(T) K_{\gamma, m}(T) \int_{0}^{t}(t-s)^{-\alpha} s^{2\left(\frac{n}{4}-\frac{1}{2}\right)-\theta-\gamma} d s \\
& =\left\{K_{\alpha, 0}(T)+C e B\left(1-\alpha, \frac{n}{4}-\frac{1}{2}\right) K_{\theta, m}(T) K_{\gamma, m}(T)\right\} t^{\frac{n}{4}-\frac{1}{2}-\alpha}
\end{aligned}
$$

for all $0<t \leq T \leq 1$. Hence (3.12) is true for $m+1$ if we take $K_{\alpha, m+1}(T)$ as

(3.14) $K_{\alpha, m+1}(T) \equiv K_{\alpha, 0}(T)+C e B\left(1-\alpha, \frac{n}{4}-\frac{1}{2}\right) K_{\theta, m}(T) K_{\gamma, m}(T)$. 
Defining $K_{m}(T) \equiv \max \left\{K_{\theta, m}(T), K_{\gamma, m}(T)\right\}$, we have by (3.14) that

$$
K_{m+1}(T) \leq K_{0}(T)+C_{*} K_{m}(T)^{2},
$$

where $C_{*}=C_{*}(n, \theta, \gamma)$ is independent of $T \in(0,1]$. Hence if

$$
K_{0}(T)<\frac{1}{4 C_{*}}
$$

then the sequence $\left\{K_{m}(T)\right\}_{m=0}^{\infty}$ is bounded as

$$
K_{m}(T) \leq \frac{1-\sqrt{1-4 C_{*} K_{0}(T)}}{2 C_{*}} \text { for all } m=0,1, \cdots
$$

It is easy to see as in the proof of Fujita-Kato [5] and Giga-Miyakawa [GM] that such a uniform bound yields a limit $\lim _{m \rightarrow \infty} v_{m}(t)=v(t)$ which is a unique solution of (3.7) with properties (3.1), (3.2) and (3.3). It remains therefore to show that we can take $T>0$ so that (3.16) is fulfilled. Indeed, since $a \in D\left(A^{\gamma}\right)$, there holds

$$
\begin{aligned}
K_{\alpha, 0}(T) & =\sup _{0<t<T} t^{\alpha-\left(\frac{n}{4}-\frac{1}{2}\right)}\left\|\widetilde{A}^{\alpha} e^{-t \widetilde{A}} a\right\| \\
& =\sup _{0<t<T} t^{\alpha-\left(\frac{n}{4}-\frac{1}{2}\right)}\left\|\widetilde{A}^{\alpha-\gamma} e^{-l \widetilde{A}} \widetilde{A}^{\gamma} a\right\| \\
& <T^{\gamma-\left(\frac{n}{4}-\frac{1}{2}\right)}\left\|\widetilde{A^{\gamma}} a\right\|
\end{aligned}
$$

for all $\gamma \leq \alpha<1$ and all $0<T \leq 1$. Since $\gamma \leq(n+2) / 8$, we have $\gamma \leq \theta$ and hence (3.18) shows that the condition (3.16) can be achieved provided

$$
T=\left(8 C_{*}\left\|\widetilde{A}^{\gamma} a\right\|\right)^{-\frac{1}{\gamma-\left(\frac{n}{4}-\frac{1}{2}\right)}} .
$$

This implies (3.4).

(2) Let us take $T=1$ in the above argument. By (3.13) we have

$$
K_{0}(1) \leq\left\|\widetilde{A}^{\frac{n}{4}-\frac{1}{2}} a\right\| .
$$

On the other hand, (3.17) shows that, according to the size of $K_{0}(1)$, we can control $\sup _{0<t<1} t^{\gamma-\left(\frac{n}{4}-\frac{1}{2}\right)}\left\|\widetilde{A}^{\gamma} u(t)\right\|\left(\leq e \sup _{0<t<1}\right.$ $\left.t^{\gamma-\left(\frac{n}{4}-\frac{1}{2}\right)}\left\|\tilde{A}^{\gamma} v(t)\right\|\right)$ arbitrarily small. Since $\sup _{0<t<1}\|u(t)\| \leq\|a\|,(3.20)$ guarantees the existence of such a constant $\mu$ as (3.5). This completes the proof of Theorem 3.1. 
Based on (3.4), we can construct a global strong solution for small initial data.

THEOREM 3.2. - Let $n=4,5$ and let $\gamma_{*}=(n+2) / 8$. There is a constant $\delta=\delta(n)>0$ such that if $a \in D\left(A^{\gamma_{*}}\right)$ satisfies

$$
\|a\|+\left\|A^{\gamma_{*}} a\right\| \leq \delta
$$

then the solution $u$ in Theorem 3.1 is global, and we may set $T=\infty$ in (3.1), (3.2) and (3.3) with $\gamma=\gamma_{*}$.

Proof. - Let $u$ be the solution on $(0, T)$ in Theorem 3.1 (1). On account of concrete characterization of the time interval for existence of the local solution such as (3.4), it suffices to show the a priori bounds of $\sup _{0<t<T}\|u(t)\|$ and $\sup _{0<t<T}\left\|A^{\gamma_{*}} u(t)\right\|$ which hold independently of $T$. Then by the usual argument of continuation, we may set $T=\infty$.

(3.3) yields that $\sup _{0<t<T}\|u(t)\| \leq\|a\|$ and hence we have only to obtain an a priori estimate of $\sup _{0<t<T}\left\|A^{\gamma_{+}} u(t)\right\|$. To this end, we shall make use of the following representation

$$
\begin{aligned}
& u(t)=e^{-t(A+1)} a+\int_{0}^{t} e^{-(t-s)(A+1)} u(s) d s \\
&-\int_{0}^{t} e^{-(t-s)(A+1)} P(u \cdot \nabla u)(s) d s \\
& \equiv u_{0}(t)+u_{1}(t)+u_{2}(t), \quad 0<t<T .
\end{aligned}
$$

Since $A$ is a non-negative self-adjoint operator in $L_{\sigma}^{2}$, there holds

$$
\left\|A^{\alpha} e^{-t(A+1)} b\right\| \leq t^{-\alpha} e^{-t}\|b\|, \quad 0 \leq \alpha \leq 1
$$

for all $b \in L_{\sigma}^{2}$ and all $t>0$. Hence we have

$$
\sup _{0<t<T}\left\|A^{\alpha} u_{0}(t)\right\| \leq\left\|A^{\alpha} a\right\| \quad \text { for } 0 \leq \alpha \leq \gamma_{*} .
$$

By (3.3) and (3.23) there holds

$$
\begin{aligned}
\left\|A^{\alpha} u_{1}(t)\right\| & \leq \int_{0}^{t}\left\|A^{\alpha} e^{-(t-s)(A+1)} u(s)\right\| d s \\
& \leq \int_{0}^{t}(t-s)^{-\alpha} e^{-(t-s)}\|u(s)\| d s \\
& \leq \sup _{0<s<T}\|u(s)\| \int_{0}^{t}(t-s)^{-\alpha} e^{-(t-s)} d s \\
& \leq \Gamma(1-\alpha)\|a\|, \quad 0 \leq \alpha<1
\end{aligned}
$$


for all $0<t<T$. Moreover, it follows from (2.6) with $\theta=n / 4-1 / 2$ that

$$
\begin{aligned}
\left\|A^{\alpha} u_{2}(t)\right\| & \leq \int_{0}^{t}\left\|A^{\alpha} e^{-(t-s)(A+1)} P(u \cdot \nabla u)(s)\right\| d s \\
& \leq \int_{0}^{t}(t-s)^{-\alpha} e^{-(t-s)}\|P(u \cdot \nabla u)(s)\| d s \\
& \leq C_{*} \int_{0}^{t}(t-s)^{-\alpha} e^{-(t-s)}\left(\left\|A^{\frac{1}{2}} u(s)\right\|^{2}+\left\|A^{\gamma_{*}} u(s)\right\|^{2}\right) d s \\
& \leq C_{*} \Gamma(1-\alpha)\left(\sup _{0<t<T}\left\|A^{\frac{1}{2}} u(t)\right\|^{2}+\sup _{0<t<T}\left\|A^{\gamma_{*}} u(t)\right\|^{2}\right)
\end{aligned}
$$

for $0<t<T, 0 \leq \alpha<1$ with $C_{*}=C_{*}(n)$ independent of $T$.

Defining $K(T) \equiv \max \left\{\sup _{0<t<T}\left\|A^{\frac{1}{2}} u(t)\right\|, \sup _{0<t<T}\left\|A^{\gamma_{*}} u(t)\right\|\right\}$, we have by (3.24), (3.25) and (3.26) that

$$
K(T) \leq \eta\left(\|a\|+\left\|A^{\gamma_{*}} a\right\|\right)+2 \eta C_{*} K(T)^{2},
$$

where $\eta=I^{\prime}\left(1-\gamma_{*}\right)\left(>I^{\top}(1 / 2)\right)$. Now we take such $\delta$ in (3.21) as

$$
0<\delta<\frac{1}{8 \eta^{2} C_{*}}
$$

Then under the assumption (3.21), we have by (3.27) that

$$
K(T) \leq \frac{1-\sqrt{1-8 \eta^{2} C_{*}\left(\|a\|+\left\|A^{\gamma_{*}} a\right\|\right)}}{4 \eta C_{*}}<\frac{1}{4 \eta C_{*}} .
$$

Since the right hand side of the above inequality is independent of $T$, we get the desired a priori estimate. This proves Theorem 3.2.

\section{DECAY OF STRONG SOLUTION}

In this section, we shall first investigate the asymptotic behavior of the global solution $u(t)$ given by Theorem 3.2, and then prove the main theorem by making use of smoothing effect for $t>0$ of the solution with $a \in D\left(A^{\frac{n}{4}-\frac{1}{2}}\right)$.

THEOREM 4.1. - The global solution $u$ in Theorem 3.2 has the following decay properties:

$$
\begin{aligned}
\left\|A^{\alpha} u(t)\right\| & =o\left(t^{-\alpha}\right) \quad \text { for } 0 \leq \alpha \leq 1, \\
\left\|\frac{d u(t)}{d t}\right\| & =o\left(t^{-1}\right)
\end{aligned}
$$


as $t \rightarrow \infty$.

This theorem will be proved in the series of the subsequent lemmata; (4.1) and (4.2) will be obtained from Lemmata 4.9 and 4.8 below, respectively. We shall follow the method established by Masuda [22]. To get sharper rates of decay as $t \rightarrow \infty$, however, we need the continuous embedding as in the proof of Lemma 2.3 and make deeper investigation into $d u(t) / d t$.

Proposition 4.2 (Masuda). - Let $0 \leq \alpha<1$ and let $g \in L^{p}\left(0, \infty ; L_{\sigma}^{2}\right)$ for some $p$ with $(1-\alpha)^{-1}<p<\infty$. Then the function $f_{\alpha}(t)$ defined by

$$
f_{\alpha}(t) \equiv \int_{0}^{t} A^{\alpha} e^{-(t-s)(A+1)} g(s) d s
$$

converges to zero as $t \rightarrow \infty$ in $L_{\sigma}^{2}$.

For the proof, see Masuda [22, Lemma 21].

LEMMA 4.3. - For the global solution $u(t)$ given by Theorem 3.2 we have

$$
\left\|A^{\frac{1}{2}} u(t)\right\| \rightarrow 0 \quad \text { as } t \rightarrow \infty
$$

Proof. - By (3.1) for $\gamma=\gamma_{*}(\equiv(n+2) / 8)$ and (3.3) with $T=\infty$, there holds

$$
\begin{aligned}
& A^{\alpha} u \in B C\left([0, \infty) ; L_{\sigma}^{2}\right) \text { for } 0 \leq \alpha \leq \gamma_{*} \\
& A^{\frac{1}{2}} u \in L^{p}\left(0, \infty ; L_{\sigma}^{2}\right) \text { for } 2 \leq p \leq \infty
\end{aligned}
$$

Let us recall the representation of $u(t)$ such as (3.22). Clearly by (3.23), we have

$$
\left\|A^{\frac{1}{2}} u_{0}(t)\right\|=\left\|A^{\frac{1}{2}} e^{-t(A+1)} a\right\| \leq t^{-\frac{1}{2}} e^{-t}\|a\| \rightarrow 0 \quad \text { as } t \rightarrow . \infty
$$

By (4.4), (4.5) and Proposition 4.2 with $p=2$,

$$
\left\|A^{\frac{1}{2}} u_{1}(t)\right\|=\left\|\int_{0}^{t} e^{-(t-s)(A+1)} A^{\frac{1}{2}} u(s) d s\right\| \rightarrow 0 \quad \text { as } t \rightarrow \infty .
$$

Taking $\theta-n / 4-1 / 2$, we have by Remark 2.6 that $F_{\theta}(u, u)(t) \in D\left(A^{\frac{\theta}{2}}\right)$ with $A^{\frac{\theta}{2}} F_{\theta}(u, u)(t)=P(u \cdot \nabla u)(t)$ for all $t \geq 0$. Hence it follows that

$$
A^{\frac{1}{2}} u_{2}(t)=\int_{0}^{t} A^{\frac{1}{2}+\frac{\theta}{2}} e^{-(t-s)(A+1)} F_{\theta}(u, u)(s) d s .
$$


Since $n / 4-\theta / 2=\gamma_{*}$, we have by Lemma $2.5,(4.4)$ and (4.5)

$$
F_{\theta}(u, u) \in L^{p}\left(0, \infty ; L_{\sigma}^{2}\right) \text { for } 2 \leq p \leq \infty .
$$

Since $\{1-(1 / 2+\theta / 2)\}^{-1}=8 /(6-n) \geq 2$, Proposition 4.2 and (4.8) yield

$$
\left\|A^{\frac{1}{2}} u_{2}(t)\right\| \rightarrow 0 \quad \text { as } t \rightarrow \infty .
$$

Now (4.3) follows from (4.6), (4.7) and (4.9).

Lemma 4.4. - For the global solution $u$ in Theorem 3.2, there exist $T_{0}>0$ and a family $\left\{f_{\alpha}(t)\right\}_{0 \leq \alpha \leq 1 / 2}$ of continuous functions on $\left[T_{0}, \infty\right)$ such that

$$
\left\|A^{\alpha} u_{h}(t)\right\| \leq f_{\alpha}(t), \quad T_{0} \leq t<\infty
$$

for all sufficiently small $h$, where $u_{h}(t) \equiv \frac{u(t+h)-u(t)}{h}$.

Proof. - Let us first note the identity

$$
(u \cdot \nabla v, w)=-(u \cdot \nabla w, v) \quad \text { for all } u, v, w \in D\left(A^{\frac{1+\alpha}{2}}\right),
$$

where $\alpha \equiv n / 6-2 / 3$. From this we have $(u \cdot \nabla v, v)=0$ for all $u, v \in D(A)$. For $u, v, w \in C_{0, \sigma}^{\infty},(4.10)$ is an immediate consequence of integration by parts. By (1.2), there holds the continuous embedding

$$
D\left(A^{\frac{1+\alpha}{2}}\right) \subset H^{1+\alpha, 2} \subset H^{1, p} \subset L^{q},
$$

where $1 / p=1 / 2-\alpha / n$ and $1 / q=1 / 2-(1+\alpha) / n$. Since $1 / p+2 / q=1$, we have by the Hölder inequality and the above embedding that

$|(u \cdot \nabla v, w)| \leq\|u\|_{q}\|\nabla v\|_{p}\|w\|_{q} \leq C\|u\|_{D\left(A^{\frac{1+\alpha}{2}}\right)}\|v\|_{D\left(A^{\frac{1+\alpha}{2}}\right)}\|w\|_{D\left(A^{\frac{1+\alpha}{2}}\right)}$

with $C=C(n)$, which implies that $(u \cdot \nabla v, w)$ is a trilinear continuous form on $D\left(A^{\frac{1+a}{2}}\right)$. On the other hand since $n=4,5$, we have $0 \leqq \alpha<1 / 2$ and hence $C_{0, \sigma}^{\infty}$ is dense in $D\left(A^{\frac{1+\alpha}{2}}\right)$ (see Fujiwara [7] and Fujita-Morimoto [6]). Now (4.10) follows from the density argument.

Since $u$ satisfies $(\mathrm{E})$,

$\dot{u}_{h}(t)+A u_{h}(t)+P\left(u_{h}(t) \cdot \nabla u(t)\right)+P\left(u(t+h) \cdot \nabla u_{h}(t)\right)=0, \quad t>|h|$,

where $\dot{u}_{h}=d u_{h} / d t$. Taking the inner product in $L_{\sigma}^{2}$ with $u_{h}$ and (4.11), we obtain from (4.10)

$$
\frac{d}{d t}\left\|u_{h}(t)\right\|^{2}+2\left\|A^{\frac{1}{2}} u_{h}(t)\right\|^{2}=2\left(u_{h}(t) \cdot \nabla u_{h}(t), u(t)\right) .
$$


By the Sobolev inequality and (2.8) with $\alpha=n / 2-2$, there holds

$$
\begin{aligned}
& \left|\left(u_{h}(t) \cdot \nabla u_{h}(t), u(t)\right)\right| \\
& \leq\left\|u_{h}(t)\right\|_{\frac{2 n}{n-2}}\left\|\nabla u_{h}(t)\right\|\|u(t)\|_{n} \\
& \leq C\left\|\nabla u_{h}(t)\right\|^{2}\left(\left\|A^{\frac{1}{2}} u(t)\right\|+\left\|A^{\frac{n}{4}-\frac{1}{2}} u(t)\right\|\right) \\
& \leq C\left\|A^{\frac{1}{2}} u_{h}(t)\right\|^{2}\left(\left\|A^{\frac{1}{2}} u(t)\right\|+\left\|A^{\frac{1}{2}} u(t)\right\|^{\frac{6-n}{n-2}}\left\|A^{\gamma_{*}} u(t)\right\|^{\frac{2(n-4)}{n-2}}\right)
\end{aligned}
$$

with $C=C(n)$ independent of $t$ and $h$. By (4.4) and Lemma 4.3, there exists $T_{0}>0$ such that

$$
\left\|A^{\frac{1}{2}} u(t)\right\|+\left\|A^{\frac{1}{2}} u(t)\right\|^{\frac{6-n}{n-2}}\left\|A^{\gamma_{+}} u(t)\right\|^{\frac{2(n-4)}{n-2}} \leq \begin{gathered}
1 \\
2 C
\end{gathered} \text { for all } t \geq T_{0} .
$$

Hence by (4.12) and (4.13) we have

$$
\frac{d}{d t}\left\|u_{h}(t)\right\|^{2}+\left\|A^{\frac{1}{2}} u_{h}(t)\right\|^{2} \leq 0 \quad \text { for all } t \geq T_{0},
$$

which yields

$$
\left\|u_{h}(t)\right\|^{2} \leq\left\|u_{h}\left(T_{0}\right)\right\|^{2} \leq\left\|\dot{u}\left(T_{0}\right)\right\|^{2}+1, \quad t \geq T_{0}
$$

for all sufficiently small $h$, where $\dot{u}=d u / d t$. Then $f_{0}(t)$ is defined by the constant function

$$
f_{0}(t) \equiv\left(\left\|\dot{u}\left(T_{0}\right)\right\|^{2}+1\right)^{\frac{1}{2}}, \quad t \geq T_{0}
$$

We shall next consider for $\alpha=1 / 2$. Taking the inner product in $L_{\sigma}^{2}$ with $A u_{h}(t)$ and (4.11), we have

$$
\text { (4.15) } \begin{aligned}
& \frac{1}{2} \frac{d}{d t}\left\|A^{\frac{1}{2}} u_{h}(t)\right\|^{2}+\left\|A u_{h}(t)\right\|^{2} \\
& \leq\left|\left(P\left(u_{h}(t) \cdot \nabla u(t)\right), A u_{h}(t)\right)\right|+\left|\left(P\left(u(t+h) \cdot \nabla u_{h}(t)\right), A u_{h}(t)\right)\right| \\
& \equiv g_{1}^{(h)}(t)+g_{2}^{(h)}(t) .
\end{aligned}
$$


Take $p$ and $q$ as $1 / p=1 / 4-1 / 2 n$ and $1 / q=1 / 4+1 / 2 n$, respectively. Then we have by $(2.8),(2.12)$ with $\alpha=n / 4-1 / 2$ and (4.4) that

$$
\begin{aligned}
& g_{1}^{(h)}(t) \\
& \quad \leq\left\|u_{h}(t)\right\|_{p}\|\nabla u(t)\|_{q}\left\|A u_{h}(t)\right\| \\
& \quad \leq C\left(\left\|A^{\frac{1}{2}} u_{h}(l)\right\|+\left\|A^{\gamma_{+}} u_{h}(l)\right\|\right)\left(\left\|A^{\frac{1}{2}} u(l)\right\|+\left\|A^{\gamma_{+}} u(l)\right\|\right)\left\|A u_{h}(l)\right\| \\
& \quad \leq C\left(\left\|A^{\frac{1}{2}} u_{h}(t)\right\|+\left\|A^{\frac{1}{2}} u_{h}(t)\right\|^{\frac{3}{2}-\frac{n^{2}}{4}}\left\|A u_{h}(t)\right\|^{\frac{n}{4}-\frac{1}{2}}\right)\left\|A u_{h}(t)\right\| \\
& \quad \leq \frac{1}{4}\left\|A u_{h}(t)\right\|^{2}+C\left\|A^{\frac{1}{2}} u_{h}(t)\right\|^{2} \\
& g_{2}^{(h)}(t) \\
& \quad \leq\|u(t+h)\|_{p}\left\|\nabla u_{h}(t)\right\|_{q}\left\|A u_{h}(t)\right\| \\
& \quad \leq C\left(\left\|A^{\frac{1}{2}} u(t+h)\right\|+\left\|A^{\gamma_{*}} u(t+h)\right\|\right) \\
& \quad\left(\left\|A^{\frac{1}{2}} u_{h}(t)\right\|+\left\|A^{\gamma_{*}} u_{h}(t)\right\|\right)\left\|A u_{h}(t)\right\| \\
& \quad \leq C\left(\left\|A^{\frac{1}{2}} u_{h}(t)\right\|+\left\|A^{\frac{1}{2}} u_{h}(t)\right\|^{\frac{3}{2}-\frac{n}{4}}\left\|A u_{h}(t)\right\|^{\frac{n}{4}-\frac{1}{2}}\right)\left\|A u_{h}(t)\right\| \\
& \leq \frac{1}{4}\left\|A u_{h}(t)\right\|^{2}+C\left\|A^{\frac{1}{2}} u_{h}(t)\right\|^{2}
\end{aligned}
$$

with $C=C(n)$ independent of $t$ and $h$. Hence it follows from (4.15) that

$$
\frac{d}{d t}\left\|A^{\frac{1}{2}} u_{h}(t)\right\|^{2}+\left\|A u_{h}(t)\right\|^{2} \leq C\left\|A^{\frac{1}{2}} u_{h}(t)\right\|^{2}, \quad t>|h| .
$$

By the Gronwall inequality, we have

$$
\begin{aligned}
\left\|A^{\frac{1}{2}} u_{h}(t)\right\|^{2} & \leq\left\|A^{\frac{1}{2}} u_{h}\left(T_{0}\right)\right\|^{2} e^{C\left(t-T_{0}\right)} \\
& \leq\left(\left\|A^{\frac{1}{2}} \dot{u}\left(T_{0}\right)\right\|^{2}+1\right) e^{C\left(t-T_{0}\right)}, \quad t \geq T_{0}
\end{aligned}
$$

for all sufficiently small $h$ with $C=C(n)$. Now the function $f_{1 / 2}(t)$ is defined by

$$
f_{\frac{1}{2}}(t) \equiv\left(\left\|A^{\frac{1}{2}} \dot{u}\left(T_{0}\right)\right\|^{2}+1\right)^{\frac{1}{2}} e^{C\left(t-T_{0}\right)}, \quad t \geq T_{0} .
$$

For $0<\alpha<1 / 2$, we have $\left\|A^{\alpha} u\right\| \leq\|u\|^{1-2 \alpha}\left\|A^{1 / 2} u\right\|^{2 \alpha}$ and hence $f_{\alpha}(t)$ can be defined by $f_{\alpha}(t)=f_{0}(t)^{1-2 \alpha} f_{1 / 2}(t)^{2 \alpha}$. This completes the proof of Lemma 4.4 .

LeMma 4.5. - Let $T_{0}>0$ be as in Lemma 4.4. Then we have

$$
\|\dot{u}(t)\|^{2}+\int_{s}^{t}\|\nabla \dot{u}(\tau)\|^{2} d \tau \leq\|\dot{u}(s)\|^{2}
$$


for all $s$ and $t$ such that $T_{0} \leq s \leq t<\infty$ and

$$
\int_{T_{0}}^{\infty}\|\dot{u}(\tau)\|^{2} d \tau<\infty
$$

Proof. - By (4.14), there holds

$$
\left\|u_{h}(t)\right\|^{2}+\int_{s}^{t}\left\|\nabla u_{h}(\tau)\right\|^{2} d \tau \leq\left\|u_{h}(s)\right\|^{2} \quad \text { for } T_{0} \leq s \leq t<\infty .
$$

Letting $h \rightarrow 0$ in the above inequality, from Lemma 4.4 and the Lebesgue convergence theorem we obtain (4.16).

Taking $\theta=n / 4-1 / 2$ in Lemma 2.5, we have by (4.4) and Remark 2.6 that $A^{\frac{\theta}{2}} F_{\theta}(u, u)(t)=P(u \cdot \nabla u)(t)$ for all $t \geq 0$. Hence the identity (E) yields

$$
\begin{aligned}
\|\dot{u}(t)\|^{2}= & -(A u, \dot{u})-(P(u \cdot \nabla u), \dot{u}) \\
= & -\left(A^{\frac{1}{2}} u, A^{\frac{1}{2}} \dot{u}\right)-\left(F_{\theta}(u, u), A^{\frac{\theta}{2}} \dot{u}\right) \\
\leq & \left\|A^{\frac{1}{2}} u\right\|\left\|A^{\frac{1}{2}} \dot{u}\right\|+\left\|F_{\theta}(u, u)\right\|\left\|A^{\frac{\theta}{2}} \dot{u}\right\| \\
\leq & \left\|A^{\frac{1}{2}} u\right\|^{2}+\left\|A^{\frac{1}{2}} \dot{u}\right\|^{2} \\
& +C\left\|A^{\frac{1}{2}} u\right\|\left(\left\|A^{\frac{1}{2}} u\right\|+\left\|A^{\gamma_{*}} u\right\|\right)\|\dot{u}\|^{1-\theta}\left\|A^{\frac{1}{2}} \dot{u}\right\|^{\theta} \\
\leq & \left\|A^{\frac{1}{2}} u\right\|^{2}+\left\|A^{\frac{1}{2}} \dot{u}\right\|^{2}+\frac{1}{2}\|\dot{u}\|^{2} \\
& +C\left\{\left\|A^{\frac{1}{2}} u\right\|\left(\left\|A^{\frac{1}{2}} u\right\|+\left\|A^{\gamma_{*}} u\right\|\right)\right\}^{\frac{2}{1+\theta}}\left\|A^{\frac{1}{2}} \dot{u}\right\|^{\frac{2 \theta}{1+\theta}} \\
\leq & \left\|A^{\frac{1}{2}} u\right\|^{2}+\left\|A^{\frac{1}{2}} \dot{u}\right\|^{2}+\frac{1}{2}\|\dot{u}\|^{2} \\
& +C\left\{\left\|A^{\frac{1}{2}} u\right\|\left(\left\|A^{\frac{1}{2}} u\right\|+\left\|A^{\gamma_{*}} u\right\|\right)\right\}^{2}+\left\|A^{\frac{1}{2}} \dot{u}\right\|^{2} \\
\leq & \left\|A^{\frac{1}{2}} u\right\|^{2}+2\left\|A^{\frac{1}{2}} \dot{u}\right\|^{2}+\frac{1}{2}\|\dot{u}\|^{2} \\
& +C\left\|A^{\frac{1}{2}} u\right\|^{4}+C\left\|A^{\gamma_{*}} u\right\|^{2}\left\|A^{\frac{1}{2}} u\right\|^{2},
\end{aligned}
$$

for all $t>0$ with $C=C(n)$, from which and (4.4) we obtain

$\|\dot{u}(t)\|^{2} \leq C\left\{\left\|A^{\frac{1}{2}} u(t)\right\|^{4}+\left(1+\sup _{\tau \geq 0}\left\|A^{\gamma_{\mu}} u(\tau)\right\|^{2}\right)\left\|A^{\frac{1}{2}} u(t)\right\|^{2}+\left\|A^{\frac{1}{2}} \dot{u}(t)\right\|^{2}\right\}$

for all $t>0$. Then (4.17) follows from (4.5) and (4.16). This completes the proof of Lemma 4.5 . 
LEMmA 4.6. - The global solution $u$ in Theorem 3.2 decays like

$$
\begin{aligned}
\|\dot{u}(t)\| & \leq C\left(t-T_{0}\right)^{-\frac{1}{2}}, \\
\left\|A^{\frac{1}{2}} u(t)\right\| & \leq C\left(t-T_{0}\right)^{-\frac{1}{1}}, \\
\|A u(t)\| & \leq C\left(t-T_{0}\right)^{-\frac{1}{2}},
\end{aligned}
$$

for all $t>T_{0}$ with $C=C\left(n, T_{0}\right)$, where $T_{0}$ is chosen as in Lemma 4.4.

Proof. - By (4.16) we have

$$
\|\dot{u}(t)\|^{2} \leq\|\dot{u}(s)\|^{2} \quad \text { for } T_{0} \leq s \leq t .
$$

Integrating this inequality on the interval $\left(T_{0}, t\right)$ with respect to $s$, we obtain from (4.17)

$$
\left(t-T_{0}\right)\|\dot{u}(t)\|^{2} \leq \int_{T_{0}}^{t}\|\dot{u}(s)\|^{2} d s \leq \int_{T_{0}}^{\infty}\|\dot{u}(s)\|^{2} d s<\infty
$$

for all $t>T_{0}$, which implies (4.18).

By (4.10) and (E) there holds $(\dot{u}(t), u(t))+(A u(t), u(t))=0$ for all $t>0$ and hence (3.3) and (4.18) yield

$$
\begin{aligned}
\left\|A^{\frac{1}{2}} u(t)\right\|^{2} & =(A u(t), u(t)) \\
& \leq|(\dot{u}(t), u(t))| \\
& \leq\|\dot{u}(t)\|\|u(t)\| \\
& \leq C\|a\|\left(t-T_{0}\right)^{-\frac{1}{2}} \quad \text { for all } t \geq T_{0}
\end{aligned}
$$

with $C=C\left(n, T_{0}\right)$, which shows (4.19).

To prove (4.20) notice that there is a constant $C_{*}=C_{*}\left(T_{0}\right)$ such that

$$
\|A u(t)\| \leq C_{*} \quad \text { for all } t \geq T_{0} .
$$

Indeed, by (2.6) with $\theta=n / 4-1 / 2$, we have (4.22) $\|A u(t)\| \leq\|\dot{u}(t)\|+\|P(u \cdot \nabla u)(t)\|$

$$
\leq\|\dot{u}(t)\|+C\left(\left\|A^{\frac{1}{2}} u(t)\right\|+\left\|A^{\gamma_{*}} u(t)\right\|\right)^{2} \quad \text { for all } t>0
$$

Hence (4.21) follows from (4.4) and (4.18). Moreover, by (4.21) and (4.22) there holds

$$
\begin{aligned}
\|A u(t)\| & \leq\|\dot{u}(t)\|+C\left\|A^{\frac{1}{2}} u(t)\right\|^{2}+C\left\|A^{\gamma_{*}} u(t)\right\|^{2} \\
& \leq\|\dot{u}(t)\|+C\left\|A^{\frac{1}{2}} u(t)\right\|^{2}+C\left(\left\|A^{\frac{1}{2}} u(t)\right\|^{2-2 \gamma_{*}}\|A u(t)\|^{2 \gamma_{*}-1}\right)^{2} \\
& \leq\|\dot{u}(t)\|+C_{\varepsilon}\left\|A^{\frac{1}{2}} u(t)\right\|^{2}+\varepsilon\|A u(t)\|^{2} \\
& \leq\|\dot{u}(t)\|+C_{\varepsilon}\left\|A^{\frac{1}{2}} u(t)\right\|^{2}+\varepsilon C_{*}\|A u(t)\|, \quad t \geq T_{0}
\end{aligned}
$$

Vol. $16, \mathrm{n}^{\circ} 5-1999$. 
for all $\varepsilon>$ with $C_{\varepsilon}$ depending only on $\varepsilon$. Taking $\varepsilon=1 / 2 C_{*}$ in the above estimate, we have

$$
\|A u(t)\| \leq C\left(\|\dot{u}(t)\|+\left\|A^{\frac{1}{2}} u(t)\right\|^{2}\right) \text { for all } t \geq T_{0}
$$

with $C=C\left(n, T_{0}\right)$. Now (4.20) follows from (4.18) and (4.19). This proves Lemma 4.6 .

Really, the global solution $u$ in Theorem 3.2 decays more rapidly than in Lemma 4.6. To obtain sharper rates of decay, we need to estimate the nonlinear term $u \cdot \nabla u$ in $L^{2}$ by means of $\left\|A^{\alpha} u\right\|$ with $\alpha \geq 1 / 2$, which differs essentially from the result of Masuda [22].

LemMa 4.7. - The global solution $u$ in Theorem 3.2 decays, in fact, like

$$
\left\|A^{\frac{1}{2}} u(t)\right\|=o\left(t^{-\frac{1}{2}}\right) \text { as } t \rightarrow \infty \text {. }
$$

Proof. - We shall make use of the representation

$$
\begin{aligned}
u(t) & -e^{-(t-T) A} u(T)-\int_{T}^{t} e^{-(t-s) A} P(u \cdot \nabla u)(s) d s \\
& \equiv I_{0}(t)+I_{1}(t)
\end{aligned}
$$

for all $0 \leq T \leq t$. By (4.19) and (4.20), there is $T_{1}>T_{0}$ such that

$$
\left\|A^{\frac{1}{2}} u(t)\right\| \leq C t^{-\frac{1}{4}}, \quad\|A u(t)\| \leq C t^{-\frac{1}{2}} \quad \text { for all } t \geq T_{1}
$$

with $C=C\left(T_{0}, T_{1}\right)$ independent of $t$. By (3.1) and Lemma 2.5 (2), we have $P(u \cdot \nabla u)(t)=A^{\theta / 2} F_{\theta}(u, u)(t)$ for all $\theta$ with $n / 2-2 \leq \theta<1$ and all $t>0$. Let us take $\theta$ as $2 / 3<\theta<1$. Then we obtain from Lemma 2.5 and (4.25)

$$
\begin{aligned}
\left\|A^{\frac{1}{2}} I_{1}(t)\right\| & \leq \int_{T}^{t}\left\|A^{\frac{1}{2}} e^{-(t-s) A} P(u \cdot \nabla u)(s)\right\| d s \\
& \leq \int_{T}^{t}\left\|A^{\frac{1}{2}+\frac{\theta}{2}} e^{-(t-s) A} F_{\theta}(u, u)(s)\right\| d s \\
& \leq C \int_{T}^{t}(t-s)^{-\frac{1}{2}-\frac{\theta}{2}}\left\|A^{\frac{1}{2}} u(s)\right\|\left(\left\|A^{\frac{1}{2}} u(s)\right\|+\left\|A^{\frac{\pi}{4}-\frac{\theta}{2}} u(s)\right\|\right) d s \\
(4.26) \quad & \leq C \int_{T}^{t}(t-s)^{-\frac{1}{2}-\frac{\theta}{2}}\left(\left\|A^{\frac{1}{2}} u(s)\right\|^{2}+\left\|A^{\frac{1}{2}} u(s)\right\|^{2-\alpha}\|A u(s)\|^{\alpha}\right) d s \\
& \leq C \int_{T}^{t}(t-s)^{-\frac{1}{2}-\frac{\theta}{2}}\left(s^{-\frac{1}{2}}+s^{-\frac{1}{2}-\frac{\alpha}{4}}\right) d s \\
& \leq C T^{-\frac{\theta}{2}}
\end{aligned}
$$


for all $t \geq T \geq T_{1}$ with $C=C\left(T_{0}, T_{1}\right)$, where $\alpha \equiv n / 2-1-\theta$. Hence by (4.24) and (4.26), there holds

$$
\begin{aligned}
\left\|A^{\frac{1}{2}} u(t)\right\| & \leq\left\|A^{\frac{1}{2}} I_{0}(t)\right\|+\left\|A^{\frac{1}{2}} I_{1}(t)\right\| \\
& \leq\left(t-T_{0}\right)^{-\frac{1}{2}}\|a\|+C T^{-\frac{\theta}{2}}, \quad T_{1}<T \leq t
\end{aligned}
$$

with $C$ independent of $T$ and $t$. Taking $T=t / 2$ in the above estimate, we have

$$
\left\|A^{\frac{1}{2}} u(t)\right\| \leq C t^{-\frac{\theta}{2}} \quad \text { for all } t \geq 2 T_{1} .
$$

Now, substituting (4.25) and (4.27) again into (4.26) we get

$$
\begin{aligned}
\left\|A^{\frac{1}{2}} I_{1}(t)\right\| & \leq C \int_{T}^{t}(t-s)^{-\frac{1}{2}-\frac{\theta}{2}}\left(s^{-\theta}+s^{-\frac{\theta}{2}(2-\alpha)} s^{-\frac{\alpha}{2}}\right) d s \\
& \leq C T^{-\frac{1}{2}(3 \theta-1)}
\end{aligned}
$$

for all $t \geq T \geq 2 T_{1}$ which yields

$$
\left\|A^{\frac{1}{2}} u(t)\right\| \leq C(t-T)^{-\frac{1}{2}}\|u(T)\|+C T^{\frac{1}{2}(3 \theta} \quad \text { 1) } \quad \text { for all } t \geq T \geq 2 T_{1}
$$

with $C=C\left(T_{0}, T_{1}\right)$. Taking $T=t / 2$ in the above estimate, we have

$$
\left\|A^{\frac{1}{2}} u(t)\right\| \leq C\left(t^{-\frac{1}{2}}\|u(t / 2)\|+t^{-\frac{1}{2}(3 \theta-1)}\right) \quad \text { for all } t \geq 4 T_{1} .
$$

Since $u$ satisfies (3.3) with $T=\infty$, we know $\lim _{t \rightarrow \infty}\|u(t)\|=0$ (see e.g., Masuda [23, Corollary 2]). Hence this estimate together with the relation $(3 \theta-1) / 2>1 / 2$ yields the desired result. This proves Lemma 4.7.

Let us improve the decay rate for $\dot{u}$.

LEMMA 4.8. - For the global solution $u$ in Theorem 3.2 we have

$$
\|\dot{u}(t)\|=o\left(t^{-1}\right) \quad \text { as } t \rightarrow \infty .
$$

Proof. - We shall make use of (4.14). To this end, we need to estimate $\left\|A^{\frac{1}{2}} u_{h}(t)\right\|$ from below. Since $u$ satisfies (E), by (4.10) there holds

$$
\begin{aligned}
\left\|u_{h}\right\|^{2} & =\left(u_{h}, u_{h}-\dot{u}\right)+\left(u_{h}, \dot{u}\right) \\
& =\left(u_{h}, u_{h}-\dot{u}\right)+\left(u_{h},-A u-P(u \cdot \nabla u)\right) \\
& =\left(u_{h}, u_{h}-\dot{u}\right)-\left(A^{1 / 2} u_{h}, A^{1 / 2} u\right)+\left(u \cdot \nabla u_{h}, u\right) \\
& \leq\left\|u_{h}\right\|\left\|u_{h}-\dot{u}\right\|+\left\|A^{1 / 2} u_{h}\right\|\left\|A^{1 / 2} u\right\|+\left(u \cdot \nabla u_{h}, u\right) .
\end{aligned}
$$


By (2.8) with $\alpha=n / 4-1$ we have

$$
\begin{aligned}
\left|\left(u \cdot \nabla u_{h}, u\right)\right| & \leq\|u\|_{4}^{2}\left\|\nabla u_{h}\right\| \\
& \leq C\left(\left\|A^{\frac{1}{2}} u\right\|^{2}+\left\|A^{\frac{n}{8}} u\right\|^{2}\right)\left\|A^{\frac{1}{2}} u_{h}\right\| \\
& \leq C\left(\left\|A^{\frac{1}{2}} u\right\|^{2}+\left(\left\|A^{\frac{1}{2}} u\right\|^{2-\frac{n}{4}}\|A u\|^{\frac{n}{4}-1}\right)^{2}\right)\left\|A^{\frac{1}{2}} u_{h}\right\| \\
& \leq C\left(\left\|A^{\frac{1}{2}} u\right\|+\left\|A^{\frac{1}{2}} u\right\|^{3-\frac{n}{2}}\|A u\|^{\frac{n}{2}-2}\right)\left\|A^{\frac{1}{2}} u\right\|\left\|A^{\frac{1}{2}} u_{h}\right\| .
\end{aligned}
$$

By Lemma 4.6 , there exists $T_{*}>T_{0}$ such that

$$
\left\|A^{\frac{1}{2}} u(t)\right\|+\left\|A^{\frac{1}{2}} u(t)\right\|^{3-\frac{n}{2}}\|A u(t)\|^{\frac{n}{2}-2} \leq \frac{1}{C} \quad \text { for all } t>T_{*}
$$

and hence we have

$$
\left|\left(u(t) \cdot \nabla u_{h}(t), u(t)\right)\right| \leq\left\|A^{\frac{1}{2}} u(t)\right\|\left\|\mid A^{\frac{1}{2}} u_{h}(t)\right\| \quad \text { for all } t>T_{*} .
$$

(4.29) and (4.30) yield

$$
\left\|A^{\frac{1}{2}} u_{h}(t)\right\|^{2} \geq \frac{\left\|u_{h}(t)\right\|^{4}}{8\left\|A^{\frac{1}{2}} u(t)\right\|^{2}}-\frac{\left\|u_{h}(t)\right\|^{2}\left\|u_{h}(t)-\dot{u}(t)\right\|^{2}}{4\left\|A^{\frac{1}{2}} u(t)\right\|^{2}}
$$

for all $t \geq T_{*}$. Substituting this estimate into (4.14), we have

$$
\frac{d\left\|u_{h}(t)\right\|^{2}}{d t}+\frac{\left\|u_{h}(t)\right\|^{4}}{8\left\|A^{\frac{1}{2}} u(t)\right\|^{2}} \leq\left\|u_{h}(t)\right\|^{2} \frac{\left\|u_{h}(t)-\dot{u}(t)\right\|^{2}}{4\left\|A^{\frac{1}{2}} u(t)\right\|^{2}}
$$

for all $t \geq T_{*}$ and all $h>0$. Set $y(t) \equiv\left\|u_{h}(t)\right\|^{2}, p(t) \equiv 8\left\|A^{1 / 2} u(t)\right\|^{2}$ and $\varepsilon_{h}(t) \equiv 2\left\|u_{h}(t)-\dot{u}(t)\right\|^{2}$. Then we have from above

$$
\frac{d y(l)}{d t}+\frac{y(l)^{2}}{p(t)} \leq \frac{\varepsilon_{h}(t)}{p(t)} \cdot y(t), \quad t \geq T_{*}
$$

which may be regarded as a differential inequality of the Bernoulli type with respect to $y(t)$. A standard calculation yields the following estimate:

$$
y(t) \leq e^{\int_{s}^{t} \frac{\varepsilon_{h}(\tau)}{p(\tau)} d \tau}\left(\frac{1}{y(s)}+\int_{s}^{t} \frac{d \tau}{p(\tau)}\right)^{-1}, \quad T_{*} \leq s \leq t
$$

This implies that

$$
\left\|u_{h}(t)\right\|^{2} \leq e^{\left.\int_{s}^{t} \frac{\varepsilon_{h}(\tau)}{8\left\|A^{1 / 2} u(\tau)\right\|^{2}}\right) d \tau}\left(\frac{1}{\left\|u_{h}(s)\right\|^{2}}+\frac{1}{8} \int_{s}^{t} \frac{d \tau}{\left\|A^{\frac{1}{2}} u(\tau)\right\|^{2}}\right)^{-1}
$$


for all $t>s \geq T_{0}$. Letting $h \rightarrow 0$ in the above, we have by Lemma 4.4 and the Lebesgue dominated convergence theorem that

$$
\|\dot{u}(t)\|^{2} \leq\left(\frac{1}{\|\dot{u}(s)\|^{2}}+\frac{1}{8} \int_{s}^{t} \frac{d \tau}{\left\|A^{\frac{1}{2}} u(\tau)\right\|^{2}}\right)^{-1}
$$

for all $t>s \geq T_{0}$. Now the desired result follows from Lemma 4.7. This completes the proof.

We shall next show (4.1).

Lemma 4.9. - For the global solution $u$ in Theorem 3.2 we have

$$
\left\|A^{\alpha} u(t)\right\|=o\left(t^{-\alpha}\right) \quad \text { for } 0 \leq \alpha \leq 1
$$

as $t \rightarrow \infty$.

Proof. - For $\alpha=0$, the assertion follows from energy decay of solutions with (3.3) (see e.g., Masuda [23, Corollary 2]). Since $\left\|A^{\alpha} u\right\| \leq$ $\|u\|^{1-\alpha}\|A u\|^{\alpha}$ for $0<\alpha<1$, it suffices to show for $\alpha=1$. By Lemmata 4.7 and 4.8 we have

$$
\|\dot{u}(t)\|=o\left(t^{-1}\right), \quad\left\|A^{\frac{1}{2}} u(t)\right\|=o\left(t^{-\frac{1}{2}}\right) \quad \text { as } t \rightarrow \infty .
$$

Applying this decay property to (4.23), we can deduce $\|A u(t)\|=o\left(t^{-1}\right)$ as $t \rightarrow \infty$.

Now Theorem 4.1 follows form Lemmata 4.8 and 4.9 .

Proof of the Main Theorem. - Let $\gamma_{*}$ and $\delta=\delta=\delta(n)$ be as in Theorem 3.2.

By Theorem 3.1 (2), there is a constant $\mu=\mu(n)$ such that if

$$
\|\dot{a}\|_{D\left(A^{\left.\frac{n}{4}-\frac{1}{2}\right)}\right.} \leq \mu
$$

then we have a unique solution $v$ of $(\mathrm{E})$ on $(0,1)$ with $\left(3.1^{\prime}\right)$ and $(3.2)$ for $T=1$ satisfying

$$
\|v(1 / 2)\|+\left\|A^{\gamma_{*}} v(1 / 2)\right\| \leq \delta .
$$

On the other hand, by Theorems 3.2 and 4.1, there exists a solution $w(t)$ of (E) on $[1 / 2, \infty)$ with $\left.w(t)\right|_{t=1 / 2}=v(1 / 2)$ satisfying (4.1) and (4.2) with $u(t)$ replaced by $v(t)$. Let us define a function $u(t)$ on $(0, \infty)$ by

$$
u(t)= \begin{cases}v(t) & \text { for } 0 \leq t \leq 1 / 2, \\ w(t) & \text { for } 1 / 2 \leq t<\infty .\end{cases}
$$

Then it is easy to verify that $u$ is a solution of (E) on $(0, \infty)$ with the desired properties. By (1.2) and (1.3) we have

$$
u \in B C\left([0, \infty) ; L^{n}\right) .
$$

and the assertion on uniqueness follows from Serrin [26] and Sohr-von Wahl [27]. 


\section{ACKNOWLEDGMENT}

This work was partially supported by the Sonderforschungsbereich 256 of Federal Republic of Germany. The first author should like to express his hearty thanks to Prof. S. Hildebrandt for his generous hospitality and support during his stay at the University of Bonn in 1996.

\section{REFERENCES}

[1] J. BERGi and J. LöFström, Interpolation Spaces. Berlin Heidelberg-New York Springer Verlag 1976.

[2] W. Borchers and T. MiYakawa, Algebraic $L^{2}$ decay for Navier-Stokes flows in exterior domains. Acta Math., Vol. 165, 1990, pp. 189-227.

[3] W. Borchers and T. MiYakawa, $L^{2}$ decay for Navier-Stokes Flows in unbounded domains, with application to exterior stationary flows. Arch. Rational Mech. Anal., Vol. 118, 1992, pp. 273-295.

[4] W. Borchers and T. MiYakaWA, On some coercive estimate for the exterior Stokes problem. Lecture Note in Math. Vol. 1530, Springer-Verlag, 1993, pp. 71-84.

[5] H. FujITA and T. Kato, On the Navier-Stokes initial value problem 1. Arch. Rational Mech. Anal., Vol. 46, 1964, pp. 269-315.

[6] H. Fujita and H. Morimoto, On fractional power of the Stokes operators. Proc. Japan Acad., Vol. 46, 1970, pp. 1141-1143.

[7] D. FUIWARA, Concrete characterization of the domains of fractional powers of some elliptic differential operators of the second order. Proc. Japan Acad., Vol. 43, 1967, pp. 82-86.

[8] G. P. Galdi and P. Maremonti, Monotonic decreasing and asymptotic behaviour of the kinetic energy for weak solutions of the Navier-Stokes equations in exterior domains. Arch. Rational Mech. Anal., Vol. 94, 1986, pp. 253-266.

[9] Y. GIGA, Domains of fractional powers of the Stokes operator in $L_{r}$, spaces. Arch. Rational Mech. Anal., Vol. 89, 1985, pp. 25!-265.

[10] Y. Giga and T. MiYakawa, Solution in $L_{r}$ of the Navier-Stokes initial value problem. Arch. Rational Mech. Anal., Vol. 89, 1985, pp. 267-281.

[11] Y. GigA and H. SoHR, On the Stokes operator in exterior domains. J. Fac. Sci. Univ. Tokyo, Sec IA, Vol. 36, 1989, pp. 103-130.

[12] J. G. HeYwood, The Navier-Stokes equations: On the existence, regularity and decay of solutions. Indiana Univ. Math. J., Vol. 29, 1980, pp. 639-681.

[13] A. InOUE, and M. Wakimoto, On existence of solutions of the Navier-Stokes equation in a time dependent domain. J. Fac. Sci. Univ. Tokyo, Sec IA, Vol. 24, 1977, pp. 103-130.

[14] H. IWASHITA, $L_{q}-L_{r}$ estimates for solutions of the nonstationary Stokes equations in an exterior domain and the Navier-Stokes initial value problems in $L_{q}$ spaces. Math. Ann., Vol. 285, 1989, pp. 265-288.

[15] S. JANSON and P. W. JONES, Interpolation between $H^{p}$ spaces: the complex method J. Funct. Anal., Vol. 48, 1982, pp. 58-81.

[16] T. KATn, $L^{p}$-solution of the Navier-Stokes equation in $R^{m}$, with applications to weak solutions. Math. Z., Vol. 187, 1984, pp. 471-480.

[17] H. Kozono and T. Ogawa, Some $L^{p}$ estimate for the exterior Stokes flow and an application to the nonstationary Navier-Stokes equations. Indiana Univ. Math. J., Vol. 41, 1992, pp. $789-808$.

[18] H. Kozono and T. Ogawa, Two-dimensional Navier-Stokes flow in unbounded domains. Math. Ann., Vol. 297, 1993, pp. 1-31. 
[19] H. Kozono and T. OGawa, Global strong solution and its decay properties for the NavierStokes equations in three dimensional domains with non-compact boundaries. Math. Z., Vol. 216, 1994, pp. 1-30.

[20] J. Leray, Sur le mouvement d'un liquids visqeux emplissant l'espace. Acta. Math., Vol. 63, 1994, pp. 193-248.

[21] P. Maremonti, Some results on the asymptotic behaviour of Hopf weak solutions to the Navier-Stokes equations in unbounded domains. Math. Z., Vol. 210, 1992, pp. 1-22.

[22] K. MASUDA, On the stability of incompressible viscous fluid motions past objects. J. Math. Soc. Japan, Vol. 27, 1975, pp. 294-327.

[23] K. MASUDA, Weak solutions of the Navier-Stokes equations. Tohoku Math. J., Vol. 36, 1984, pp. 623-646.

[24] T. MIYAKAWA, On nonstationary solutions of the Navier-Stokes equations in an exterior domain. Hiroshima Math. J., Vol. 12, 1982, pp. 115-140.

[25] T. MIYAKAWA and H. SoHr, On energy inequality, smoothness and large time behavior in $L^{2}$ for weak solutions of the Navier-Stokes equations in exterior domains. Math. Z., Vol. 199, 1988, pp. 455-478.

[26] J. Serrin, The initial value problem for the Navier-Stokes equations. R. E. Langer ed., University of Wisconsin Press, Madison, 1963, pp. 69-98.

[27] H. SOHR and W. Von WAHL, On the singular set and the uniqueness of weak solutions of the Navier-Stokes equations. Manuscripta Math., Vol. 49, 1984, pp. 27-59.

[28] H. Tanabe, Equations of evolutions. London: Pitman 1979.

[29] S. UKaI, $\Lambda$ solution formula for the Stokes equation in $R_{+}^{\prime \prime}$. Comm. Pure Appl. Math., Vol. 40, 1987, pp. 611-621.

$[30] \mathrm{W}$. von WAHL, The equations of Navier-Stokes and abstract parabolic equations. Braunschweig Wiesbaden: Vieweg 1985.

(Manuscript received on February 27, 1996;

Revised version received June 18, 1997.) 